\section{FRI0209 RECOMMENDATIONS FOR ACQUISITION AND CONSIDERATIONS FOR INTERPRETATION OF MRI OF THE SPINE AND SACROILIAC JOINTS IN THE INVESTIGATION OF AXIAL SPONDYLOARTHRITIS IN THE UK}

T. Bray, A. Jones, M.A. Hall-Craggs, A. Bennett, P.G. Conaghan, A. Grainger, R. Hodgson, C. Hutchinson, M. Leandro, P. Mandl, D. McGonagle, P. O'Connor R. Sengupta, M. Thomas, A. Toms, N. Winn, H. Marzo-Ortega, P.M. Machado. MRI Working Group, British Society for Spondyloarthritis, BRITSA, UK

Background: The use of magnetic resonance imaging (MRI) has been instrumental in the early recognition and characterisation of axial spondyloarthritis (axSpA). However, a recent survey in the UK showed that there is diverse practice in the use of MRI and limited knowledge of MRI features suggestive of axSpA among radiologists.

Objectives: To develop clinical practice recommendations for the acquisition and interpretation of MRI of the spine and sacroiliac joints (SIJs) in the investigation of axSpA through a collaboration between rheumatologists and radiologists.

Methods: A working group comprising 9 rheumatologists and 9 musculoskeletal radiologists with an interest in axSpA was established. The EULAR standardised operating procedures were followed. ${ }^{2}$ Two working group meetings were held, the first to define the scope of the exercise and the second to review the results of the Systematic Literature Review that informed the recommendations. An anonymised Delphi process was used to formulate the final set of recommendations. The level of evidence and strength of recommendation was added to the recommendations. The level of agreement by working group members was assessed using a numerical rating scale.

Results: A total of 2 overarching principles and 7 recommendations were formulated (figure 1). The first 3 recommendations address the MRI acquisition protocol, namely anatomical areas to be scanned and sequences to be used. The remaining 4 recommendations address the interpretation of active and structural lesions of the spine and SIJs.

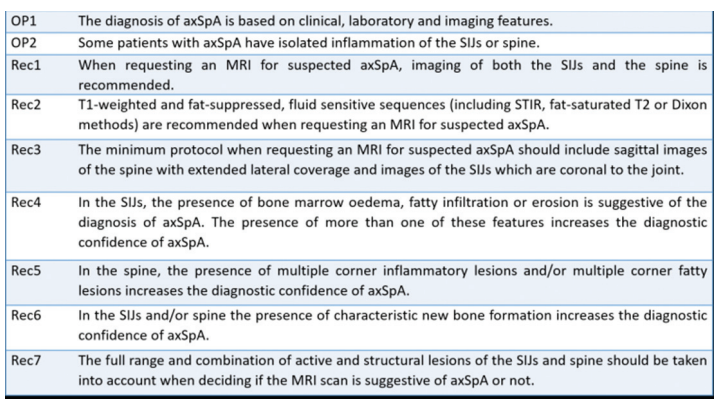

Abstract FRI0209 - Figure 1. Overarching principles (OP) and recommendations (Rec)

Conclusions: A UK joint rheumatology and radiology consensus on the most appropriate MRI acquisition protocol and interpretation of images in the investigation of axSpA was achieved. This consensus will help standardise practices and ensure prompt and effective patient management in the diagnosis and treatment of $\operatorname{axSpA}$.

\section{REFERENCES:}

[1] Bennett, et al. J Rheumatol 2017.

[2] van der Heijde, et al. Ann Rheum Dis 2015.

Acknowledgements: This work was financially supported by the British Society for Spondyloarthritis (BRITSpA).

Disclosure of Interest: None declared

DOI: 10.1136/annrheumdis-2018-eular.5806

\section{FRI0210 EXTRA-ARTICULAR MANIFESTATIONS ARE ASSOCIATED WITH WORSE QUALITY OF LIFE AND CLINICAL OUTCOME IN PATIENTS WITH AXIAL SPONDYLOARTHRITIS}

R. van der Meer ${ }^{1}$, S. Arends ${ }^{1,2}$, S. Kruidhof ${ }^{1}$, R. Bos ${ }^{2}$, H. Bootsma ${ }^{1}$, F. Wink ${ }^{2}$, A. Spoorenberg ${ }^{1,2} .{ }^{1}$ Rheumatology and Clinical Immunology, University of Groningen, University Medical Center Groningen, Groningen; ${ }^{2}$ Rheumatology, Medical Center Leeuwarden, Leeuwarden, Netherlands

Background: In the 1960s, the association between axial spondyloarthritis (SpA) and extra-articular manifestations (EAMs), acute anterior uveitis (AAU), inflammatory bowel disease (IBD) and psoriasis, was first reported. Still, knowledge of axial SpA disease outcome associated with the development of EAMs is scarce.

Objectives: To investigate the prevalence and 4 year incidence of EAMs and to explore associations of newly developed EAMs with disease outcome after 4 years of follow-up in a large cohort of axial SpA patients.

Methods: All consecutive patients fulfilling the modified New York criteria for AS or the ASAS criteria for axial SpA from the prospective observational Groningen Leeuwarden Axial Spondyloarthritis (GLAS) cohort with a baseline visit between November 2004 and December 2011 were included in the analyses. Baseline and follow-up data of EAMs from the GLAS database were verified in the medica records. EAMs were only recorded and used for analyses when a description of the diagnosis by an ophthalmologist, gastroenterologist or dermatologist was present. Prevalence and 4 year incidence of EAMs was calculated and comparative analyses regarding disease outcome was performed.

Results: 421 axial SpA patients were included with a mean age of $43.4 \pm 12.5$ years, $65 \%$ were male, mean symptom duration was $17.4 \pm 11.7$ years, $78 \%$ were HLA-B27 positive, mean ASDAS was $3.3 \pm 1.1$ and $66 \%$ started TNF- $\alpha$ inhibitors at baseline. Of the 421 patients, $132(31.4 \%)$ had a positive history of one or more EAMs: 104 (24.8\%) AAU, 40 (9.5\%) IBD, and 18 (4.3\%) psoriasis. Of the 362 patients with 4 year follow-up data, $57(15.7 \%)$ patients developed an EAM: 48 (13.3\%) patients AAU, of which $13(3.6 \%)$ had a first episode, $7(1.9 \%)$ patients developed IBD, and $3(0.8 \%)$ patients developed psoriasis.

Patients who developed a new EAM without a history of EAMs at baseline had higher ASQoL (mean 10.0 vs. 5.9, $\mathrm{p}=0.001$ ), larger occiput to wall distance (median 6.3 vs. 2.0, $\mathrm{p}=0.021$ ) and also the modified Schober test was more limited (mean 12.6 vs. 13.6, $\mathrm{p}=0.014$ ) after 4 years of follow-up. The difference found for BASFI was not statistically significant (mean 4.4 vs. $3.4, p=0.12$ ).

Conclusions: The prevalence rates of EAMs in our cohort are similar as found in other axial SpA studies. The 4 year incidence of EAMs was relatively low, possibly due to the relatively large proportion of patients starting TNF- $\alpha$ inhibitors at baseline. However, these axial SpA patients showed worse quality of life and clinica outcome than patients without a newly developed EAM

Disclosure of Interest: R. van der Meer: None declared, S. Arends Grant research support from: Pfizer, S. Kruidhof: None declared, R. Bos: None declared H. Bootsma: None declared, F. Wink Consultant for: Abbvie, A. Spoorenberg Grant/research support from: Pfizer, Abbvie, Consultant for: Pfizer, Abbvie, MSD, UCB, Novartis

DOI: 10.1136/annrheumdis-2018-eular.6334

\begin{tabular}{|l|l}
\hline FRI0211 & ASSOCIATION OF NEUROPATHIC-LIKE PAIN \\
CHARACTERISTICS WITH CLINICAL AND \\
RADIOGRAPHIC FEATURES IN PATIENTS WITH \\
ANKYLOSING SPONDYLITIS
\end{tabular}

S.-H. Lee, H.-R. Kim, K.-A. Lee. Konkuk University Medical Center, Seoul, Korea, Republic Of

Background: Ankylosing spondylitis (AS) is a chronic progressive inflammatory disorder that mainly involves the axial skeleton and causes chronic back pain. It is not unusual for patients with AS to have symptoms similar to neuropathic pain There were several studies showing that various rheumatic diseases, including rheumatoid arthritis, primary Sjogren syndrome, and fibromyalgia, had neuropathic pain components. However, the existence of neuropathic pain in patients with AS has not been well investigated. The painDETECT questionnaire (PD-Q) is a relatively simple and self-administered screening tool for determining neuropathic pain and has high sensitivity, specificity, and positive predictive accuracy values.

Objectives: The aim of this study was to investigate the neuropathic pain component in patients with AS using PD-Q, and to assess the relation between neuropathic pain and disease characteristics of AS.

Methods: A single-centre prospective study was performed on 105 patients. The patients with AS completed three questionnaires: PD-Q. Beck depression inventory (BDI), and Euro Quality of life (EQ-5D) questionnaires. Patients were classified into three groups according to the PD-Q scores: nociceptive pain (NoP (score $<13$ ), mixed pain (MP) (score 13-18), and neuropathic (NeP) (score >18) pain. Fifteen patients (14.3\%) were classified in the NeP group, 22 patients $(21.0 \%)$ in the MP group, and 68 patients $(64.7 \%)$ in the NoP group. The questionnaires and clinical and radiographic findings were analysed.

Results: Patients with NeP and MP scored worse on Bath ankylosing spondylitis disease activity index (BASDAI), BDI, modified Stoke Ankylosing Spondylitis Spine Score, pain-visual analogue scale (VAS), EQ-5L index, and showed an increased prevalence of enthesitis and peripheral arthritis (table 1). There were no differences in objective inflammatory markers. PD-Q scores positively correlated with pain-VAS, BASDAI, BDI, and inversely correlated with EQ-5D index (figure 1). Presence of enthesitis, BDI, age, and pain VAS score independently associated with PD-Q scores. 